\title{
Fear of COVID-19 and Stress-Relieving Practices Among Social Media Users, Makkah Region, Saudi Arabia
}

Ehab A. Abo-Ali ${ }^{1}$, Ahmed H. Mousa ${ }^{2}$, Mentulla W. Omar ${ }^{2}$, Shayma S. Al-Rubaki ${ }^{2}$, Wessam A. Ghareeb ${ }^{3}$, Sameh Zaytoun ${ }^{4}$

1. Community Medicine Department, Faculty of Medicine, University of Tanta, Tanta, EGY 2. Medicine and Surgery, Batterjee Medical College, Jeddah, SAU 3. Neuro-Psychiatry Department, Faculty of Medicine, University of Tanta, Tanta, EGY 4. Department of Community Medicine, Faculty of Medicine, South Valley University, Qena, EGY

Corresponding author: Ahmed H. Mousa, ahmadhafez9926@gmail.com

\section{Abstract \\ Background}

The ongoing COVID-19 pandemic and its associated consequences can trigger feelings of fear, concern, and anxiety among the population, leading to unfavorable consequences on mental health. This study aimed to assess fear of COVID-19 and stress-relieving practices among social media users in the Makkah region, Saudi Arabia.

\section{Methods}

A cross-sectional analytic study was conducted among 532 adults inhabiting the Makkah region of Saudi Arabia over a period of one month, from June 15 to July 15, 2020. A predesigned, self-administered questionnaire, including assessments of fear of COVID-19 and stress-relieving practices, was used for data collection.

\section{Results}

The mean Fear of COVID-19 Scale score was $17.3 \pm 5.21$ out of 35. Individuals aged 30-49 years and married individuals had higher mean scores ( $18.4 \pm 5.20$ and $18.4 \pm 5.29$, respectively) compared to other groups $(\mathrm{p}<0.05)$. Additionally, individuals with histories of anxiety and depression, individuals suffering from chronic diseases, and those who did not exercise regularly had higher levels of fear compared to other groups $(\mathrm{p}<0.05)$. Practicing religious and spiritual rituals was the most commonly adopted stress-relieving practice among study participants (68.6\%).

\section{Conclusion}

Review began 05/24/2021 Review ended 06/11/2021 Published 06/21/2021

\section{() Copyright 2021}

Abo-Ali et al. This is an open access article distributed under the terms of the Creative Commons Attribution License CC-BY 4.0., which permits unrestricted use, distribution, and reproduction in any medium, provided the original author and source are credited.
Adults in Saudi Arabia have considerable levels of fear of COVID-19. Special attention is recommended for highly susceptible groups. Additionally, mental health education programs are recommended for the promotion of the community's psychological resilience in such a global crisis. Spiritual aspects should be included in such mental health education programs.

\section{Categories: Infectious Disease, Public Health}

Keywords: fear of covid-19, adults, saudi arabia, covid-19 outbreak, stress-relieving

\section{Introduction}

The emergence of the novel coronavirus disease 2019 (COVID-19), caused by the severe acute respiratory syndrome coronavirus 2 (SARS-CoV-2) and first reported in December 2019 in the city of Wuhan of Hubei Province in China, has been a major point of global concern. The World Health Organization declared the COVID-19 outbreak a public health emergency of international concern on January 30, 2020, and a global pandemic on March 11, 2020 [1,2]. As of the time of writing this article (November 8, 2020), more than 49 million cases of COVID-19 have been confirmed in over 200 countries, with the number of confirmed deaths approaching 1.5 million [3].

In Saudi Arabia, the number of confirmed cases of COVID-19 is 350,229, with 5,525 deaths and 336,966 recoveries. This represents a low mortality rate of $1.58 \%$ and an impressive recovery rate of $96.21 \%$ as of November 8, 2020 [4,5]. In line with international infection control guidelines, the government of Saudi Arabia promptly implemented necessary preventive measures in workplaces, restaurants, mosques, markets, and community gatherings across the country, including social distancing, wearing masks, practicing hand hygiene, lockdowns, travel restrictions, voluntary self-quarantine, online education, and working from home $[6]$. 
The ongoing COVID-19 pandemic and its associated consequences can trigger feelings of fear, concern, and anxiety among the population. A study including 1,304 participants from 75 cities in Turkey found a strong positive association between the pandemic and fear among the population, leading to unfavorable consequences such as depression, anxiety, psychological distress, and poor mental health [1]. A new term, "coronaphobia," is being used to delineate fear of COVID-19 [7]. A systematic review of 19 studies from eight countries described the high rates of anxiety, depression, post-traumatic stress disorder, psychological distress, and stress among people during the COVID-19 pandemic [8]. Repercussions of extreme fear can include issues such as social withdrawal, stigmatization, and social discrimination and can also end with suicide, as with cases reported in India and Bangladesh [1,7,9-11]. Another study found a positive impact of wearing face masks on the physical and mental health of the population in comparison with a community that discouraged face masks [12]. Populations vulnerable to increased levels of fear include the elderly, pregnant women, and patients with preexisting psychiatric illnesses [13].

In these extraordinary circumstances resulting in unusual challenges for everyone, people must adopt coping strategies against fear and stress in an attempt to maintain physical and mental stability and wellness. Higher stress levels can lead to anxiety and disturbed sleeping and eating patterns and, subsequently, worsening physical and mental health conditions [14]. Although coping strategies can vary from person to person, recommendations from the Saudi Center for Disease Prevention and Control include decreasing time spent watching or reading the news, self-educating using reliable resources, participating in self-relaxation, practicing hobbies, communicating with others, exercising, and practicing healthy eating [15].

While a multitude of ongoing studies concerning the physical manifestation, treatment, and vaccination of COVID-19 are emerging, there are relatively few studies investigating the effects of the pandemic and related situations on the mental health of the population. This study aimed to assess fear of COVID-19 and stress-relieving practices among social media users in the western region of Saudi Arabia.

\section{Materials And Methods Study design and setting}

This was a cross-sectional, analytic study in Saudi Arabia conducted over a period of one month (June 15 to July 15, 2020).

\section{Study population and sampling technique}

All adults who were aged 18 years and above, living in Makkah Region, Saudi Arabia, and agreed to participate in the study were eligible for recruitment. The minimum sample size, calculated using a level of confidence of $95 \%$, expected prevalence of $50 \%$, and precision of 0.05 -was found to be 384 . For increased accuracy, the study's sample size was increased to 532. To comply with the physical distancing rules in response to the COVID-19 pandemic, convenient and snowball sampling techniques were adopted for recruitment of study participants through online invitations on various social media platforms.

\section{Study tool}

Data were obtained using a predesigned online questionnaire. The survey was available in both Arabic and English. For each participant, the following information was collected: (a) sociodemographic, occupational, and health characteristics and relevant family history; (b) Fear of COVID-19 Scale score (this was developed and validated by Ahorsu et al. [9] in March 2020, and its Arabic version was validated by Alyami et al. [16] in May 2020. This scale is composed of seven statements [e.g., "I am most afraid of Corona," "It makes me uncomfortable to think about Corona," "My hands become clammy when I think about Corona," "I am afraid of losing my life because of Corona”]. For each statement, every participant was asked to express agreement on a five-point Likert scale, with "1" indicating strong disagreement and " 5 " indicating strong agreement. Each patient's total score was obtained by summation of the points for all seven responses. The scale of scores ranged between 7 and 35. Higher scores indicated higher levels of fear of COVID-19); (c) stressrelieving practices (this part of the questionnaire included statements about encouraged/proposed stressrelieving practices as recommended by the $\mathrm{CDC}$, such as "taking a break from news about the pandemic" and "talking with people you trust about your concerns"); the authors added an additional question about "practicing religious and spiritual rituals," as this is expected to be of high relevance in religious communities. For each statement, the participants were asked to indicate the frequency at which they had adopted these practices in the prior month. The questioner was tested and proved to be valid and reliable.

\section{Ethical considerations}

Approval from the Ethics and Scientific Committees (UB-RES-2020-0035) of Batterjee Medical College was obtained before initiation of the study. Informed consent was obtained online from each participant; the aim of the study was clearly explained, and selecting the "agree to participate" icon was required before proceeding to the questionnaire items. Data were collected anonymously, and the confidentiality of collected data was guaranteed. 


\section{Cureus}

\section{Data analysis}

The collected data were statistically analyzed using Statistical Package for Social Sciences (SPSS) software, version 23 (IBM, Chicago, Armonk, NY). Categorical variables were presented as numbers and percentages. Numerical variables were presented as means \pm standard deviations. Testing for statistically significant differences between the subgroups was carried out. Comparing mean scores between two groups and more than two groups was carried out using student's t-test and analysis of variance (ANOVA), respectively. The level of significance was set at $\mathrm{p}<0.05$.

\section{Results}

The current study included 532 participants from the western region of Saudi Arabia. Nearly two-thirds of the participants $(n=338 ; 63.5 \%)$ were aged between 18 and 29 years. The majority were females $(n=380$; $71.4 \%)$ and individuals residing in cities $(n=514 ; 96.6 \%)$. Approximately two thirds $(n=335 ; 63.0 \%)$ were unmarried, and more than half $(\mathrm{n}=302 ; 56.8 \%)$ were Saudi citizens. Over three-fourths of the respondents $(n=412 ; 77.4 \%)$ perceived their social level as "moderate," and the majority $(n=507 ; 95.3 \%)$ were living with their families or flatmates. Most of the participants had a college degree or above $(n=442 ; 83.1 \%)$ and were employed ( $n=426 ; 80.1 \%)$. More than two-thirds of study participants $(n=363 ; 68.2 \%)$ encountered minimal social engagement in daily activities. Most of the respondents were nonsmokers ( $\mathrm{n}=448 ; 84.2 \%)$, had no history of anxiety or depression $(n=442 ; 83.0 \%)$, had family histories of chronic conditions $(n=338 ; 63.5 \%)$, and did not exercise regularly $(\mathrm{n}=350 ; 65.8 \%)$ (Table 1$)$.

Gender

Male

Residence

City

$514(96.6)$

Village

Marital Status

Married

Unmarried (single, divorced, widow...)

$335(63.0)$

Nationality

Saudi

Non Saudi

$230(43.2)$

Perceived Social Level

High

Living Arrangement

Educational Level

High school and below 


\section{Cureus}

Employment

Employed

$426(80.1)$

Unemployed or retired

$106(19.9)$

Nature of usual daily activities

With minimal social engagement (remotely or office work)

$363(68.2)$

With social engagement (field work)

$169(31.8)$

Smoking (Current)

Yes

No

448 (84.2)

History of Anxiety or Depression

Yes

$90(17.0)$

No

$442(83.0)$

Present or family history of chronic conditions

Yes

$338(63.5)$

No

$194(36.5)$

Regular exercise

Yes

$182(34.2)$

No

$350(65.8)$

TABLE 1: Sociodemographic, occupational and health characteristic of social media users, Western Region, Saudi Arabia ( $\mathrm{N}=532$ )

The highest mean fear scores were noted in response to the items "It makes me uncomfortable to think about Corona" and "I'm most afraid of Corona" (with mean fear scores of 3.3 \pm 1.08 and 3.1 \pm 0.97 , respectively). The lowest mean fear scores were found in response to the items "I cannot sleep because I'm worrying about getting Corona" and "My hands become clammy when I think about Corona," with mean scores of $1.7 \pm 0.90$ and $1.6 \pm 0.87$, respectively. The mean Fear of COVID-19 Scale score was $17.3 \pm 5.21$ out of 35 (Table 2).

Item

1. I am most afraid of Corona

2. It makes me uncomfortable to think about Corona

3. My hands become clammy when I think about Corona

$1.6 \pm 0.87$

4. I am afraid of losing my life because of Corona

$2.6 \pm 1.18$

5. When I watch news and stories about Corona on social media, I become nervous or anxious

$3.0 \pm 1.09$

6. I cannot sleep because I'm worrying about getting Corona.

$1.7 \pm 0.90$

7. My heart races or palpitates when I think about getting Corona.

$2.0 \pm 1.09$

Total fear of COVID-19 scale items

$17.3 \pm 5.21$

TABLE 2: Scores of social media users' responses to Fear of COVID-19 Scale examples in western region, Saudi Arabia $(n=532)$

The mean score recorded for the middle age group (30-49 years) was $18.4 \pm 5.20$, which was higher than the 


\section{Cureus}

scores of the lower age group (18-29 years) and the older age group ( $\geqslant 50$ years) $(16.8 \pm 5.07$ and $16.5 \pm 5.56$, respectively). The differences between all three age groups were statistically significant $(\mathrm{p}<0.05)$. Moreover, significantly higher mean fear scores were related to marital status; married participants revealed higher mean scores than unmarried ones (18.4 \pm 5.29 and $16.7 \pm 5.06$, respectively). Comparable mean fear scores were observed for male and female participants ( $16.8 \pm 5.36$ and $17.5 \pm 5.13$, respectively), with no statistically significant difference $(p>0.05)$. No significant differences in mean fear scores were present according to residency, nationality, perceived social levels, living arrangements, and educational levels ( $p>0.05$ for all) (Table 3).

\begin{tabular}{|c|c|c|c|}
\hline \multirow{2}{*}{ Socio-demographic characteristics } & \multicolumn{2}{|c|}{ Fear of COVID-19 scale scores } & \multirow{2}{*}{ t-test/ANOVA p value } \\
\hline & No. & Mean $\pm S D$ & \\
\hline \multicolumn{4}{|l|}{ Age group } \\
\hline $18-29$ & 338 & $16.8 \pm 5.07$ & \multirow{3}{*}{$<0.001^{*}$} \\
\hline $30-49$ & 144 & $18.4 \pm 5.20$ & \\
\hline 50 and above & 50 & $16.5 \pm 5.56$ & \\
\hline \multicolumn{4}{|l|}{ Gender } \\
\hline Male & 152 & $16.8 \pm 5.36$ & \multirow{2}{*}{0.139} \\
\hline Female & 380 & $17.5 \pm 5.13$ & \\
\hline \multicolumn{4}{|l|}{ Residence } \\
\hline City & 514 & $17.4 \pm 5.18$ & \multirow{2}{*}{0.086} \\
\hline Village & 18 & $15.3 \pm 5.64$ & \\
\hline \multicolumn{4}{|l|}{ Marital status } \\
\hline Married & 197 & $18.4 \pm 5.29$ & \multirow{2}{*}{$<0.001^{*}$} \\
\hline Unmarried & 335 & $16.7 \pm 5.06$ & \\
\hline \multicolumn{4}{|l|}{ Nationality } \\
\hline Saudi & 302 & $17.2 \pm 5.07$ & \multirow{2}{*}{0.663} \\
\hline Non Saudi & 230 & $17.4 \pm 5.38$ & \\
\hline \multicolumn{4}{|l|}{ Perceived social level } \\
\hline High & 95 & $17.1 \pm 5.10$ & \multirow{3}{*}{0.143} \\
\hline Moderate & 412 & $17.2 \pm 5.05$ & \\
\hline Low & 25 & $19.3 \pm 7.56$ & \\
\hline \multicolumn{4}{|l|}{ Living arrangement } \\
\hline With family or flat mates & 507 & $17.3 \pm 5.18$ & \multirow{2}{*}{0.658} \\
\hline Alone & 25 & $16.8 \pm 5.76$ & \\
\hline \multicolumn{4}{|l|}{ Educational level } \\
\hline High school and below & 90 & $17.4 \pm 5.91$ & \multirow{2}{*}{0.916} \\
\hline College degree and higher & 442 & $17.3 \pm 5.06$ & \\
\hline
\end{tabular}

TABLE 3: Fear of COVID-19 scale scores among social media users, western region, Saudi Arabia, in relation to socio-demographic characteristics

There were no statistically significant differences between mean fear scale scores with regard to employment status, nature of daily activities, or smoking habits ( $p>0.05$ for all). However, significantly higher mean fear scores were associated with other health-related parameters. Participants with histories of 


\section{Cureus}

anxiety or depression had significantly higher mean fear scores compared to those who did not have histories of such conditions ( $16.2 \pm 5.32$ and $14.4 \pm 4.14$, respectively). Participants with present or family histories of chronic conditions demonstrated significantly higher mean fear scores compared to those who did not suffer from or have family histories of such conditions (17.8 \pm 5.53 and $16.5 \pm 4.52$, respectively; $\mathrm{p}<0.05)$. Additionally, participants who did not exercise regularly demonstrated higher mean fear scores than those who did exercise regularly (17.7 \pm 5.20 and $16.6 \pm 5.21$, respectively; $p<0.05$ ) (Table 4$)$.

\begin{tabular}{|c|c|c|c|}
\hline \multirow{2}{*}{ Occupational and health characteristics } & \multicolumn{2}{|c|}{ Fear of COVID-19 scale scores } & \multirow{2}{*}{ t-test/ANOVA p-value } \\
\hline & No. & Mean \pm SD & \\
\hline \multicolumn{4}{|l|}{ Employment } \\
\hline Employed & 426 & $17.2 \pm 5.17$ & \multirow{2}{*}{0.440} \\
\hline Unemployed or retired & 106 & $17.7 \pm 5.37$ & \\
\hline \multicolumn{4}{|l|}{ Nature of usual daily activities } \\
\hline With minimal social engagement (remotely or office work) & 363 & $17.5 \pm 5.11$ & \multirow{3}{*}{0.389} \\
\hline With social engagement (field work) & 169 & $17.0 \pm 5.44$ & \\
\hline \multicolumn{3}{|l|}{ Smoking (Current) } & \\
\hline Yes & 84 & $18.1 \pm 5.03$ & \multirow{2}{*}{0.113} \\
\hline No & 448 & $17.1 \pm 5.23$ & \\
\hline \multicolumn{4}{|l|}{ History of anxiety or depression } \\
\hline Yes & 90 & $16.2 \pm 5.32$ & \multirow{2}{*}{$<0.001^{*}$} \\
\hline No & 442 & $14.4 \pm 4.14$ & \\
\hline \multicolumn{4}{|l|}{ Present or family history of chronic conditions } \\
\hline Yes & 338 & $17.8 \pm 5.53$ & \multirow{2}{*}{$0.005^{\star}$} \\
\hline No & 194 & $16.5 \pm 4.52$ & \\
\hline \multicolumn{4}{|l|}{ Regular exercise } \\
\hline Yes & 182 & $16.6 \pm 5.21$ & \multirow{2}{*}{$0.031^{\star}$} \\
\hline No & 350 & $17.7 \pm 5.20$ & \\
\hline
\end{tabular}

TABLE 4: Fear of COVID-19 scale score among social media users, western region, Saudi Arabia, in relation to occupational and health characteristics $(n=532)$

The majority of study participants $(\mathrm{n}=365 ; 68.6 \%)$ stated that their most common method of coping with stress was "practicing religious and spiritual rituals." "Self-care" was found to be the second most commonly practiced stress-relieving strategy $(\mathrm{n}=285 ; 53.6 \%)$. Conversely, the least-practiced coping strategy was found to be "connection with others" ( $\mathrm{n}=180 ; 34 \%)$ (Table 5). 


\section{Cureus}

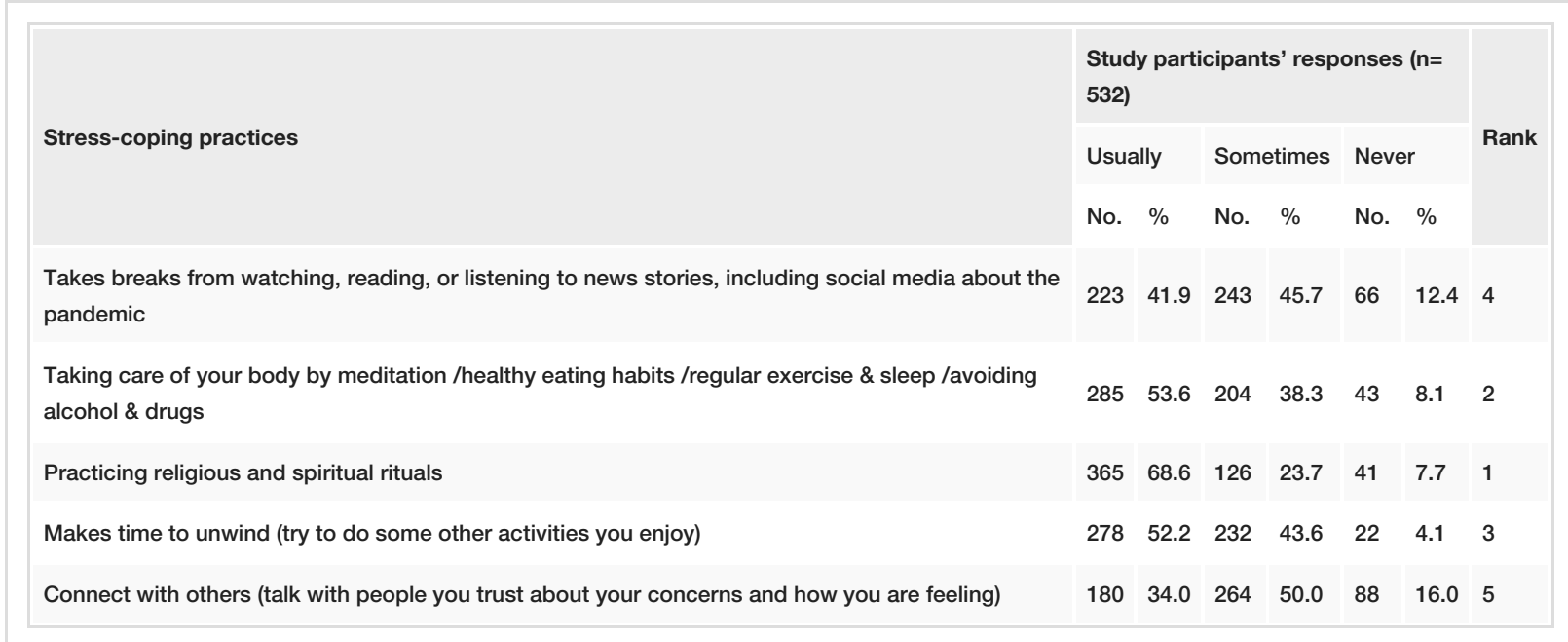

TABLE 5: Stress-coping practices in response to fear of COVID-19 among social media users, western region, Saudi Arabia $(n=532)$

\section{Discussion}

The COVID-19 pandemic has placed an overwhelming burden on healthcare systems and economies around the world. This global pandemic can provoke feelings of fear and concern among the population due to the highly contagious nature of the virus, its associated health complications, and the economic and psychological repercussions of the preventative measures imposed around the world [1].

In this study, the majority of survey respondents were females. This female response dominance may be explained by the fact that females are generally more motivated to participate in online surveys.This is in accordance with similar studies in Turkey and Eastern Europe $[1,17]$. However, marginally male-dominant responses were found in other studies $[9,16]$. Although female respondents exhibited higher levels of fear than male respondents, this difference was not significant. On the other hand, multiple studies in Eastern Europe, Israel, and the USA declared that females had higher levels of fear of COVID-19 [17-19]. Interestingly, respondents aged between 30 and 49 years revealed significantly higher levels of fear than younger and older age groups. This could be explained by a higher sense of responsibility in this stage of life. Furthermore, a study by Leo and Trabucchi revealed heightened levels of fear among Italian senior citizens, intensified by feelings of isolation, loneliness, and loss of hope due to multiple preexisting chronic diseases, demoralization, and social negligence [20]. However, in line with religious beliefs and traditions in Saudi Arabia, younger family members take care of the financial and health requirements of the elderly. This leads to feelings of security and serenity despite the high risk of health complications among the elderly. The present study found a remarkable association between married individuals and increased fear of COVID-19. This is in agreement with a study by Fitzpatrick et al., potentially indicating increased concern for the health and well-being of loved ones in this population [19].

No significant relationship was found between fear and social level or nationality. This can be attributed to the Saudi governmental efforts in treating all COVID-19 patients equally and free of charge, irrespective of their nationality or social level [21]. Meanwhile, a study in the USA showed increased fear among individuals of certain races and citizens born in other countries. Additionally, another study in Israel revealed a higher level of fear among individuals of low socioeconomic levels [18,19]. Additionally, living arrangements seemed to have no substantial relationship with fear of COVID-19; people living with family may have a sense of unity and security, while individuals who live alone might also feel safe due to the decreased risk of infecting family members. Furthermore, no evident association was found between educational level and fear of COVID-19. Among people with lower education, there is a lower level of proper knowledge about the outbreak and its effects, leading to a false sense of safety. Conversely, people with higher education are more likely to be familiar with the relevant evolving information and safety protocols, leading to a sense of safety.

The current study revealed no significant association between fear of COVID-19 and employment status. This is in agreement with the results of a similar study conducted in China [22]. One potential explanation is that employees who returned to work after the reduction of lockdown restrictions were aware of safety precautions, which enhanced their psychological resilience. Various preventive measures were announced to the population (e.g., wearing face masks and frequently sanitizing hands) [23]. Furthermore, unemployed or retired individuals showed nearly the same level of fear of COVID-19 since they are expected to experience lower levels of social interaction during the pandemic. Similarly, no association was found between fear of COVID-19 and the nature of practiced daily activities, either with minimal or evident social engagement. 
Though smoking is a factor highly associated with complications from COVID-19, the present study showed no association between fear of COVID-19 and smoking habits. This finding is in line with results from a previous study conducted in Vietnam, which revealed that smoking was a stress reliever among medical students, especially during stressful times [24].

The present study showed a significant association between fear of COVID-19 and history of anxiety and depression. Similarly, a previous study in Brazil showed that anxiety and depression can affect individuals who are most likely exposed to high rates of stress and are on the frontlines with respect to pathogens, such as individuals in healthcare professions [25]. Additionally, other studies conducted in China revealed high rates of stress symptoms and anxiety among doctors and nurses [26,27].

The current study demonstrated a significant relationship between fear of COVID-19 and associated chronic illnesses. This result is supported by a study previously conducted in Turkey [28]. This may be anticipated, especially with the emphasis of World Health Organization, that individuals who suffer from chronic illnesses are more likely to contract COVID-19 with higher mortality rates [28]. In addition, patients who are frequently treated with immunosuppressive agents and cytotoxic drugs tend to be immunocompromised and more susceptible to infections. People with systemic lupus erythematosus are considered a vulnerable population for SARS-CoV-2 infections and COVID-19. Both systemic lupus erythematosus and COVID-19 have been shown to manifest multi-organ complications, such as interstitial pneumonia, cytopenia, arthralgia, myocarditis, and hemophagocytic lymphohistiocytosis [1,29].

Additionally, the present study revealed a significant association between lack of regular exercise and increased fear of COVID-19. Frequent physical exercise is associated with the maintenance of good physical and mental health and is considered an effective mechanism of coping with stress [7].

The present study revealed that "practicing religious and spiritual rituals" was the most frequently adopted stress-relieving practice among adults in Saudi Arabia. This finding is not surprising since the study was conducted in a mostly religious community. Conversely, a study conducted in the USA revealed that the population has directed their thoughts to extreme pessimism and hopelessness, with more suicidal ideations, alcohol, and drug abuse during the COVID-19 crisis [2]. Moreover, another significant strategy for coping with stress during the pandemic is taking care of the body by way of meditation, healthy eating habits, regular exercise and sleep, and avoidance of alcohol and drugs. This is supported by another study in India that showed how such strategies can promote the mental health of individuals during these stressful times [3]. At the very least, stress-relieving practices included staying in touch with friends and family members using online platforms and social media.

\section{Strengths and limitations}

This study has the strength of being the first to explore and assess the fear of COVID-19 and stress-relieving practices in the community of Saudi Arabia. The non-probability sampling technique is a limitation of the study but was adopted due to the hindering effect of lockdown during the study period. The bias of this nonprobability sample was reduced by enlarging the sample size. Additionally, the study only recruited subjects who had access to social media platforms.

\section{Conclusions}

Considering the results of the present study, we can conclude that adults in Saudi Arabia have been experiencing fear of COVID-19 to a considerable degree. Particular groups, such as middle-aged, married, those with a history of anxiety and depression, subjects suffering from (or with a family history of) chronic diseases, and persons neglecting regular exercise reported higher levels of fear. The most commonly adopted stress-relieving practice among the study participants was "practicing religious and spiritual rituals". Special attention is recommended for the care of the aforementioned susceptible groups, and mental health education programs should be initiated for the promotion of the psychological resilience of the community. Spiritual aspects should be included in the construction of such health education programs. A populationbased study is recommended for addressing the magnitude of the problem on a wider spectrum.

\section{Additional Information \\ Disclosures}

Human subjects: Consent was obtained or waived by all participants in this study. Batterjee Medical College issued approval RES-2020-0035. Dear Researchers, We are pleased to inform you that your Research proposal Res-2020-0035 has been approved, with an emphasis to the following comments: - Inform participants of the nature of the research, their part in it and obtain their consent. - Ethical Practices: minimize harms and risks and maximize benefits; respect human dignity. $\cdot$ Maintain confidentiality of the personal data of the participants. The participants must have the right to withdraw at any time. Please Keep updating us regarding the status of your Research. Best wishes of success with your Research. Best Regards, Safiah Khalid Hussain Labani Research Co-ordinator Batterjee Medical College . Animal subjects: All authors have confirmed that this study did not involve animal subjects or tissue. Conflicts of interest: In compliance with the ICMJE uniform disclosure form, all authors declare the following: Payment/services 
info: All authors have declared that no financial support was received from any organization for the submitted work. Financial relationships: All authors have declared that they have no financial relationships at present or within the previous three years with any organizations that might have an interest in the submitted work. Other relationships: All authors have declared that there are no other relationships or activities that could appear to have influenced the submitted work.

\section{Acknowledgements}

The authors thank the study participants for their participation and their time spent completing the questionnaire.

\section{References}

1. Satici B, Gocet-Tekin E, Deniz ME, Satici SA: Adaptation of the Fear of COVID-19 Scale: its association with psychological distress and life satisfaction in Turkey. Int J Ment Health Addict [IN PRESS]. 2020, 1-9. 10.1007/s11469-020-00294-0

2. Rolling updates on coronavirus disease (COVID- 19): Updated 31 July . (2020). Accessed: June 20, 2021: https://www.who.int/emergencies/diseases/novel-coronavirus-2019/events-as-they-happen.

3. WHO Coronavirus (COVID-19) dashboard. (2021). Accessed: June 20, 2021: https://covid19.who.int/

4. COVID 19 dashboard: Saudi Arabia. (2021). Accessed: June 20, 2021: https://covid19.moh.gov.sa/.

5. Daily Updates - Public Health Authority . (2021). Accessed: June 20, 2021: https://covid19.cdc.gov.sa/dailyupdates/.

6. Community \& Public - Public Health Authority . (2021). Accessed: June 20, 2021: https://covid19.cdc.gov.sa/community-public/.

7. Dubey S, Biswas P, Ghosh R, et al.: Psychosocial impact of COVID-19. Diabetes Metab Syndr. 2020, 14:77988. 10.1016/j.dsx.2020.05.035

8. Xiong J, Lipsitz O, Nasri F, et al.: Impact of COVID-19 pandemic on mental health in the general population: A systematic review. J Affect Disord. 2020, 277:55-64. 10.1016/j.jad.2020.08.001

9. Ahorsu DK, Lin CY, Imani V, Saffari M, Griffiths MD, Pakpour AH: The Fear of COVID-19 Scale: development and initial validation. Int J Ment Health Addict. 2020, 1-9. 10.1007/s11469-020-00270-8

10. Goyal K, Chauhan P, Chhikara K, Gupta P, Singh MP: Fear of COVID 2019: First suicidal case in India! . Asian J Psychiatr. 2020, 49:101989. 10.1016/j.ajp.2020.101989

11. Mamun MA, Griffiths MD: First COVID-19 suicide case in Bangladesh due to fear of COVID-19 and xenophobia: Possible suicide prevention strategies. Asian J Psychiatr. 2020, 51:102073. 10.1016/j.ajp.2020.102073

12. Wang C, Chudzicka-Czupała A, Grabowski D, et al.: The association between physical and mental health and face mask use during the COVID-19 pandemic: A comparison of two countries with different views and practices. Front Psychiatry. 2020, 11:569981. 10.3389/fpsyt.2020.569981

13. Rajkumar RP: COVID-19 and mental health: A review of the existing literature . Asian J Psychiatr. 2020, 52:102066. 10.1016/j.ajp.2020.102066

14. Coping with stress. (2021). Accessed: June 20, 2021: https://www.cdc.gov/coronavirus/2019-ncov/daily-lifecoping/managing-stress-anxiety.html.

15. Preventive guide for mental and social health. (2021). Accessed: June 20, 2021: https://covid19.cdc.gov.sa/community-public/mental-health.

16. Alyami M, Henning M, Krägeloh CU, Alyami H: Psychometric evaluation of the Arabic version of the Fear of COVID-19 Scale [IN PRESS] . Int J Ment Health Addict. 2020, 1-14. 10.1007/s11469-020-00316-X

17. Reznik A, Gritsenko V, Konstantinov V, Khamenka N, Isralowitz R: COVID-19 fear in Eastern Europe: validation of the Fear of COVID-19 Scale. Int J Ment Health Addict. 2020, 1-6. 10.1007/s11469-020-00283-3

18. Tzur Bitan D, Grossman-Giron A, Bloch Y, Mayer Y, Shiffman N, Mendlovic S: Fear of COVID-19 scale: psychometric characteristics, reliability and validity in the Israeli population. Psychiatry Res. 2020, 289:113100. 10.1016/j.psychres.2020.113100

19. Fitzpatrick KM, Harris C, Drawve G: Fear of COVID-19 and the mental health consequences in America . Psychol Trauma. 2020, 12:S17-21. 10.1037/tra0000924

20. de Leo D, Trabucchi M: COVID-19 and the fears of Italian senior citizens. Int J Environ Res Public Health. 2020, 17:3572. 10.3390/ijerph17103572

21. Alshammari TM, Altebainawi AF, Alenzi KA: Importance of early precautionary actions in avoiding the spread of COVID-19: Saudi Arabia as an Example. Saudi Pharm J. 2020, 28:898-902. 10.1016/i.jsps.2020.05.005

22. Wang C, Pan R, Wan X, et al.: A longitudinal study on the mental health of general population during the COVID-19 epidemic in China. Brain Behav Immun. 2020, 87:40-8. 10.1016/j.bbi.2020.04.028

23. Tan W, Hao F, McIntyre RS, et al.: Is returning to work during the COVID-19 pandemic stressful? A study on immediate mental health status and psychoneuroimmunity prevention measures of Chinese workforce. Brain Behav Immun. 2020, 87:84-92. 10.1016/i.bbi.2020.04.055

24. Nguyen HT, Do BN, Pham KM, et al.: Fear of COVID-19 Scale-associations of its scores with health literacy and health-related behaviors among medical students. Int J Environ Res Public Health. 2020, 17:4164. 10.3390/ijerph17114164

25. Ornell F, Schuch JB, Sordi AO, Kessler FHP: "Pandemic fear" and COVID-19: mental health burden and strategies. Braz J Psychiatry. 2020, 42:232-5. 10.1590/1516-4446-2020-0008

26. Kang L, Li Y, Hu S, et al.: The mental health of medical workers in Wuhan, China dealing with the 2019 novel coronavirus. Lancet Psychiatry. 2020, 7:e14. 10.1016/S2215-0366(20)30047-X

27. Huang JZ, Han MF, Luo TD, Ren AK, Zhou XP: Mental health survey of medical staff in a tertiary infectious disease hospital for COVID-19 [article in Chinese]. Zhonghua Lao Dong Wei Sheng Zhi Ye Bing Za Zhi. 2020, 38:192-5. 10.3760/cma.j.cn121094-20200219-00063 


\section{Cureus}

28. Bakioğlu F, Korkmaz O, Ercan H: Fear of COVID-19 and positivity: mediating role of intolerance of uncertainty, depression, anxiety, and stress. Int J Ment Health Addict. 2020, 1-14. 10.1007/s11469-02000331-y

29. Yen EY, Singh RR: Brief report: Lupus-an unrecognized leading cause of death in young females: A population-based study using nationwide death certificates, 2000-2015. Arthritis Rheumatol. 2018, 70:12515. 10.1002/art.40512 